\title{
Construction of the Prediction Model for Locally Advanced Rectal Cancer Following Neoadjuvant Chemoradiotherapy Based on Pretreatment Tumor-Infiltrating Macrophage-Associated Biomarkers
}

This article was published in the following Dove Press journal: OncoTargets and Therapy

\author{
Xing Liu' \\ Shuping Zheng ${ }^{2}$ \\ Yong Peng ${ }^{3}$ \\ Jinfu Zhuang $\mathbb{B D}^{4}$ \\ Yuanfeng Yang ${ }^{4}$ \\ Yunlu $X u^{2}$ \\ Guoxian Guan (D) \\ 'Department of Colorectal Surgery, \\ Fujian Medical University Union Hospital, \\ Fuzhou, People's Republic of China; \\ ${ }^{2}$ Public Technology Service Center, Fujian \\ Medical University, Fuzhou, People's \\ Republic of China; ${ }^{3}$ Department of \\ General Surgery, Nanjing BenQ Medical \\ Center, The Affiliated BenQ Hospital of \\ Nanjing Medical University, Nanjing, \\ People's Republic of China; ${ }^{4}$ Department \\ of Colorectal Surgery, The First Affiliated \\ Hospital of Fujian Medical University, \\ Fuzhou, People's Republic of China
}

Correspondence: Guoxian Guan Department of Colorectal Surgery, Fujian Medical University Union Hospital, 29

Xinquan Road, Fuzhou, 35000I, Fujian,

People's Republic of China

Tel +86 59I-83357896

Email fjxhggx@163.com

Yunlu Xu

Public Technology Service Center, Fujian Medical University, I Xuefubei Road,

Fuzhou City, 35000I, Fujian, People's

Republic of China

Tel +86 59l-22862408

Email xuyunlu3578@fjmu.edu.cn
Purpose: To assess the value of macrophage-related biomarkers (CD163, CD68, MCSF, and CCL2) for predicting the response to neo-chemoradiotherapy (NCRT) and the prognosis of locally advanced rectal cancer (LARC).

Methods: We enrolled 191 patients who underwent neoadjuvant chemoradiotherapy and radical resection between 2011 and 2015. Tumor tissues were collected before NCRT with a colonoscope and post-surgery and were subjected to immunohistochemical analysis.

Results: The expression levels of macrophage-related biomarkers (CD163, CD68, MCSF, and CCL2) were lower in the pathological complete response (pCR) group when compared with the non-pCR group (all $\mathrm{P}<0.05$ ). Based on $\mathrm{X}$-tile plots, we divided the tumors in two groups and found that lower pre-NCRT/post-surgical CD163, CD68, MCSF, CCL2 scores correlated with improved DFS. Cox regression analysis demonstrated that pre-NCRT CD163 $(\mathrm{HR}=1.008,95 \%$ CI $1.003-1.013, P=0.003)$ and MCSF $(\mathrm{HR}=2.187,95 \%$ CI $1.343-3.564$, $P=0.002$ ) scores were independent predictors of DFS. Based on Cox multivariate analysis, we constructed a risk score model with a powerful ability to predict pCR in LARC patients. Moreover, $\mathrm{COX}$ regression analysis was performed to explore the role of the risk score in LARC patients. The results demonstrated that tumor size $(\mathrm{HR}=1.291, P=0.041)$, worse pathological TNM stage $(\mathrm{HR}=1.789, P=0.005$, and higher risk score $(\mathrm{HR}=1.084, P<0.001)$ were significantly associated with impaired disease-free survival. Based on the above results, a nomogram and decision curve analysis were generated.

Conclusion: The expression levels of macrophage-related biomarkers CD163, CD68, MCSF, and CCL2 were associated with chemoradiotherapy resistance and prognosis in LARC patients following NCRT. A risk score model was constructed which could be used to predict LARC outcome.

Keywords: rectal cancer, neoadjuvant chemoradiotherapy, risk, prognosis

\section{Introduction}

Macrophages are a population of innate myeloid cells that are released from the bone marrow as immature monocytic precursors. ${ }^{1,2}$ In response to different environment stimuli, macrophages which have migrated to peripheral tissues can differentiate into two polarization states: ${ }^{3}$ the classically activated M1 subtype, which activates the immune system, ${ }^{4}$ and the alternatively activated M2 subtype, which 
dampens immune responses. ${ }^{5}$ Tumor-associated macrophages (TAM) are usually M2-like macrophages which act as pro-tumor cells in several types of cancers, such as head and neck, bladder, hepatocellular, breast and colorectal cancers. ${ }^{2,5-10}$ Moreover, TAMs not only directly provide structural support for cancer development, but also secrete growth factors, cytokines and chemokines; eg, transforming growth factor $\beta$ (TGF- $\beta$ ), vascular endothelial growth factor (VEGF), M-CSF and chemokine (C-C motif ligand), which participate in tumor growth, metastasis and drug resistance. ${ }^{11-14}$ However, the function of TAMs and secreted molecules in locally advanced rectal cancer (LARC) patients undergoing neoadjuvant chemoradiotherapy (NCRT) is still unclear.

NCRT combined with total mesorectal excision (TME) has become the standard of care for LARC. This approach effectively reduces tumor size and stage, increases tumor resectability, preserves the sphincter, and results in better local tumor control. ${ }^{15-17}$ However, each LARC patient has a different response to NCRT, and approximately $15 \%$ to $45 \%$ of LARC patients show resistance to NCRT. ${ }^{18}$ Unfortunately, the mechanisms which explain resistance to NCRT are still unclear and there are no reliable biomarkers to predict clinical outcome. Previous studies have demonstrated that high density of TAMs is associated with worse response to radiotherapy and chemotherapy in several cancer types. ${ }^{19-22}$ Additionally, the expression levels of secreted molecules in the tumor micro-environment are associated with TAM density and with the response to radiotherapy and chemotherapy. Thus, it is essential to understand the relationship between TAM density, levels of locally secreted molecules and resistance to NCRT in LARC patients. Analysis of the most reliable M1 and M2 macrophage markers and of locally secreted molecules in LARC patients is still incomplete.

To address these gaps, the present study aimed to explore the relationship between TAM density, levels of locally secreted molecules and resistance to NCRT in LARC patients in terms of NCRT response and survival outcome, and to construct predictive nomograms.

\section{Patients and Methods}

Patient Eligibility, Treatment Protocol and Follow-Up

We enrolled LARC patients who underwent NCRT and radical resection between 2011 and 2015. Tumor tissues were collected before NCRT with a colonoscope and postsurgery and were used for immunohistochemical analysis. Patient inclusion criteria, treatment protocol and follow up were as described in our previous study. ${ }^{23}$ Briefly, preoperative long-course radiation consisted of a total dose of 45 Gy to the pelvis. Concurrent chemotherapy was initiated on the first day of radiotherapy using one of two chemotherapeutic regimens: 5FU plus oxaliplatin (FOLFOX) or capecitabine plus oxaliplatin (CapeOX). Patients were followed up until death or the cut-off date of December 31, 2019. This study was approved by the Institutional Review Board of Fujian Medical University Union Hospital $(2,013,051)$. And all patients provided written informed consent for the scientific use of the clinical tissue samples and clinical data.

\section{Definitions}

Tumor response to NCRT was evaluated according to the pathological tumor regression grade (TRG) ${ }^{24}$ that is, TRG 0 , no residual tumor cells (also known as pathological complete response, pCR); TRG 1, single cells or small groups of tumor cells; TRG 2, residual tumor cells with desmoplastic response; and TRG 3, minimal evidence of tumor response.

\section{Immunohistochemical Analysis}

CD68 (ab213363, Abcam, USA) and CD163 (ab182422, Abcam, USA) staining was performed to count the number of tumor-associated macrophages and M2 type macrophages, respectively. Levels of macrophage colonystimulating factor (MCSF) (ab233387, Abcam, USA) and CCL2 (bs-20201R, Bioss, China) were measured in specimens obtained before and after NCRT in 191 LARC patients using the immunohistochemical streptavidin-biotin complex method. ${ }^{25}$ Phosphate-buffered saline was used as the negative control The total number of tumor-associated macrophages was determined based on CD68 staining, while the number of M2-type macrophages was determined based on CD163. The CD68 and CD163 scores were basing on the positive macrophage cells which were calculated on the fields were randomly selected in five directions (up, center, down, left, and right) under high magnification (400). The MCSF and CCL2 was scored by semi-quantitative analysis, and the fields were randomly selected in five directions (up, center, down, left, and right) under high magnification $(\times 400)$. The color was determined based on the intensity score as follows: 0 (no staining), 1 (light yellow), 2 (brown), and 3 (deep brown). The percentage of positive cells was 
scored as $0(<5 \%), 1(5-25 \%), 2(25-50 \%), 3(50-75 \%)$, and $4(>75 \%)$. The mean value was calculated for each case with the aforementioned scoring methods and the final score was obtained by multiplying these two scores $0-3$, for negative group; 4-12, for positive group. All analyses were performed in a double-blind manner. We have already added the above sentences in the revised manuscript. ${ }^{26}$ All analyses were performed in a double-blinded manner.

\section{Statistical Analysis}

Statistical analyses were performed using SPSS version 23.0 and R software, version 3.5.1. The optimal cut-off points for pre-NCRT CD163 and pre-NCRT CD68 counts were calculated and determined by using the X-tile program, based on minimal $\mathrm{p}$ values from log-rank $\chi^{2}$ statistics in terms of DFS. ${ }^{27}$ Survival outcomes were assessed using the KaplanMeier method and Log rank test. Cox proportional hazards and risk factor models were performed to identify risk factors for OS and DFS using the R software. Based on cut-off values determined by ROC analysis, patients were divided into high-risk and low-risk groups. Kaplan-Meier curves and Cox regression analysis were performed to compare DFS risk between high-risk and low-risk groups. The performance of the model was evaluated by time-dependent ROC analysis. $\mathrm{P}<0.05$ was considered statistically significant.

\section{Results}

\section{Cutoff Values for Pre-NCRT CDI63 and CD68}

A total of 191 LARC patients (127 males and 64 females) were eligible for our analysis. Since the pre-NCRT CD68 and M2-type macrophage (CD163) scores constitute continuous variables, the X-tile program was utilized to identify the optimal cut-off points reflecting the greatest actuarial survival difference. As shown in Figure 1A and B, and Supplementary Figure S1, X-tile plots were constructed and used to identify 309 and 200 as optimal cutoff values for CD68 and CD163, respectively. Based on the above cut-off points for CD68 and CD163, we divided the entire cohort into low and high subgroups in terms of OS and DFS.

\section{Association of Pre/Post-Surgical CDI63, CD68, MCSF, CCL2 Scores with Survival and Ability to Predict PCR in NCRT \\ Patients}

Higher pre/post-surgical CD163, CD68, MCSF, CCL2 scores were associated with worse prognosis in LARC patients following NCRT. The 3-year OS rates for patients with low pre-NCRT CD163, CD68, MCSF, CCL2 scores were $88.7 \%, 88.1 \%, 88.1 \%$, and $86.4 \%$, respectively. These values were significantly lower in the high pre-NCRT CD163, CD68, MCSF, CCL2 groups: 50.0\%, 64.9\%, $60.8 \%$, and $70.7 \%$, respectively $(P<0.01$, Figure $1 \mathrm{~A}$; $P<0.01$, Figure 1B; $P<0.01$, Figure $1 \mathrm{C}$; and $P=0.12$, Figure 1D). The 3-year OS rates for patients with low post-surgical CD163, CD68, MCSF, CCL2 scores were 89.6\%, 90.1\%, $88.8 \%$, and $92.3 \%$, respectively. These values were significantly higher than those observed in the high post-surgical CD163, CD68, MCSF, CCL2 groups: 79.4\%, 78.6\%, 80.4\%, and $77.2 \%$, respectively $(P=0.01$, Figure $1 \mathrm{E} ; P=0.04$, Figure $1 \mathrm{~F} ; P=0.10$, Figure $1 \mathrm{G}$; and $P=0.01$, Figure $1 \mathrm{H})$.

Notably, lower pre-NCRT CD163, CD68, MCSF, CCL2 scores correlated with improved DFS. The 3-year
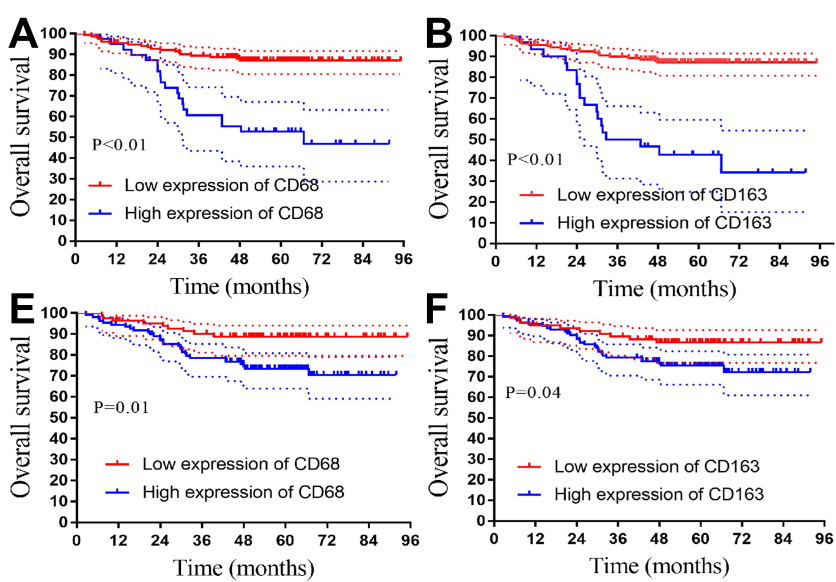
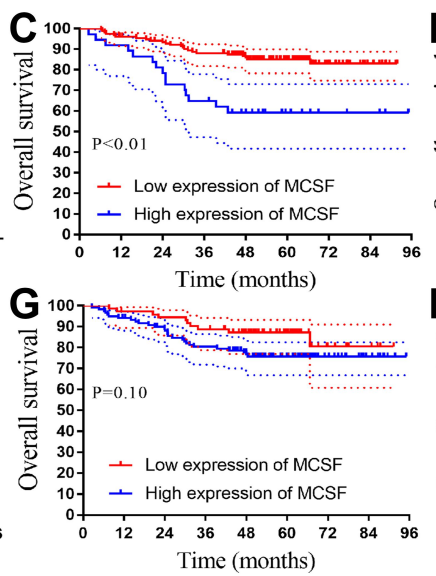

Figure I Kaplan-Meier analysis of OS based on TAMs and macrophage-related biomarkers. (A-D) Kaplan-Meier analysis of OS based on pre-NCRT CDI63, CD68, MCSF, CCL2 scores. (E-H) Kaplan-Meier analysis of OS based on post-surgical CDI63, CD68, MCSF, CCL2 scores. 
DFS rates for the low pre-NCRT CD163, CD68, MCSF, CCL2 groups were $86.5 \%, 85.1 \%, 84.6 \%, 80.1 \%$, respectively, values that were significantly higher than those observed in the high pre-NCRT CD163, CD68, MCSF, CCL2 groups: $39.3 \%, 71.0 \%, 55.4 \%$, and $75.2 \%$, respectively $(P<0.01, P<0.01, P<0.01, P=0.61)$, as shown in Figure 2A-D. Moreover, we found a similar result in the post-surgical CD163, CD68, MCSF, CCL2 groups. The 3-year DFS rates for the low post-NCRT CD163, CD68, MCSF, CCL2 groups were 85.5\%, 87.6\%, 85.8\%, 88.3\%, respectively. These values were significantly higher than those observed in the high post-NCRT CD163, CD68, MCSF, CCL2 groups: $73.8 \%, 71.6 \%, 75.1 \%$, and $72.7 \%(P=0.01, P=0.07, P=0.02, P<0.01)$, as shown in Figure 2E-H.

pCR is considered a short-term endpoint of NCRT. We analyzed the expression of macrophage-related biomarkers in the pCR and non-pCR groups, and explored the ability of macrophage-related biomarkers to predict $\mathrm{pCR}$ in LARC patients receiving NCRT (Figure 3A). As shown in Figure 3B-E, pre-NCRT CD163, CD68, MCSF, and CCL2 scores were lower in the pCR group than in the non-pCR group (all $P<0.01)$. Moreover, ROC analysis was performed to analyze the predictive ability of pre-NCRT CD163, CD68, MCSF, and CCL2 scores. The results demonstrated that pre-NCRT CD163 (AUC $=0.77, \mathrm{P}<0.01$, Figure 3G), pre-NCRT CD68 (AUC $=0.66, \quad \mathrm{P}<0.01, \quad$ Figure $3 \mathrm{~F}$ ), pre-NCRT MCSF (AUC $=0.66, \mathrm{P}<0.01$, Figure $3 \mathrm{H}$ ), and pre-NCRT CCL2 (AUC $=0.67, \mathrm{P}<0.01$, Figure 3I) scores showed a powerful ability to predict $\mathrm{pCR}$.
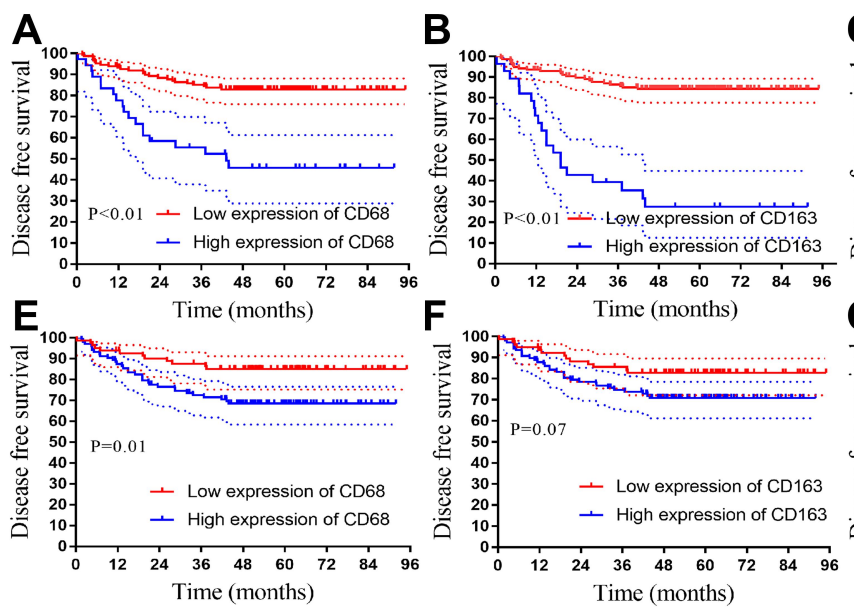

\section{Prognostic Value of \\ Macrophage-Associated Markers and Calculation of the Risk Score}

To explore the prognostic significance of pre-NCRT and post-surgical macrophage-associated markers on OS and DFS in LARC patients, we performed COX regression analysis. Univariate analysis revealed that pre-NCRT CD163 $(P<0.001)$, pre-NCRT CD68 $(P<0.001)$, preNCRT MCSF $(P<0.001), \quad$ post-surgical CD163 ( $P=0.002)$, post-surgical CD68 $(P=0.005)$, post-surgical $\operatorname{MCSF}(P=0.003)$, and post-surgical CCL2 $(P=0.001)$ scores were independently associated with DFS in LARC patients following NCRT and TME (Table 1). Multivariate Cox regression demonstrated that preNCRT CD163 (HR=1.008, 95\% CI 1.003-1.013, $P=0.003)$, pre-NCRT MCSF $\quad(\mathrm{HR}=2.187, \quad 95 \% \quad$ CI 1.343-3.564, $\quad P=0.002$ ), and post-surgical CCL2 $(\mathrm{HR}=1.494,95 \%$ CI $1.017-2.194, P=0.041)$ scores were independent predictors of DFS following NCRT, as shown in Table 1.

Univariate analysis revealed that pre-NCRT CD163 $(P<0.001)$, pre-NCRT CD68 $(P<0.001), \quad$ pre-NCRT MCSF $(P<0.001)$, pre-NCRT CCL2 $(P=0.042)$, postsurgical CD163 $(P=0.001), \quad$ post-surgical CD68 $(P=0.001)$, post-surgical MCSF $(P=0.042)$, and postsurgical CCL2 $(P=0.010)$ scores were independently associated with OS in LARC patients following NCRT and TME (Table 2). Multivariate Cox regression demonstrated that pre-NCRT CD163 (HR $=1.005,95 \%$ CI $1.001-1.011$, $P=0.049)$, and pre-NCRT MCSF $(\mathrm{HR}=2.445,95 \% \mathrm{CI}$
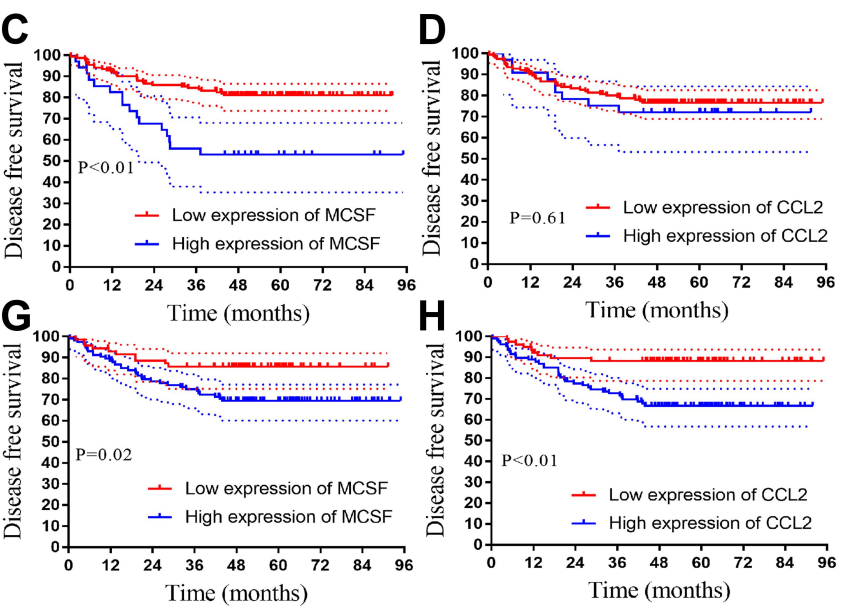

Figure 2 Kaplan-Meier analysis of DFS based on TAMs and macrophage-related biomarkers. (A-D) Kaplan-Meier analysis of DFS based on pre-NCRT CDI63, CD68, MCSF, CCL2 scores. (E-H) Kaplan-Meier analysis of DFS based on post-surgical CDI63, CD68, MCSF, CCL2 scores. 

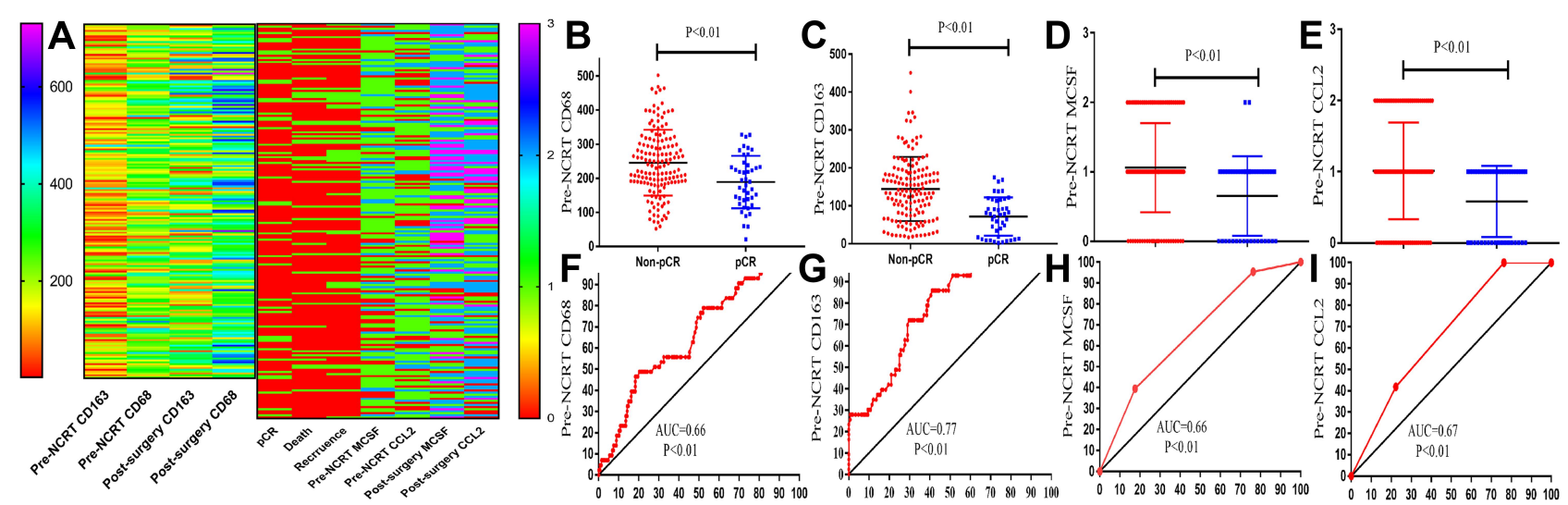

Figure 3 Association of pre-NCRT TAMs and macrophage-related biomarkers with PCR. (A) Heat map of pre-NCRT TAM scores, macrophage-related biomarker levels and clinical parameters. The left Y-axis were the CDI63 and CD68 scores and the right Y-axis were the MCSF and CCL2 scores. (B-E) Pre-NCRT TAMs were lower in the PCR group when compared with the non-pCR group (all $P<0.0 I$ ). ( $F-I)$ ROC analysis demonstrating that pre-NCRT CDI63 (AUC=0.77, P $<0.01$ ), pre-NCRT CD68 $(A \cup C=0.66, P<0.01$ ), pre-NCRT MCSF (AUC=0.66, P<0.0I), and pre-NCRT CCL2 (AUC=0.67, P<0.0I) scores had a powerful ability to predict $p C R$.

1.389-4.303, $P=0.002$ ) scores were independent predictors of OS following NCRT, as shown in Table 2.

Based on the OS and DFS Cox regression results, we selected pre-NCRT CD163 and pre-NCRT MCSF expression values to construct a risk factor model, as follows: Risk score $=0.0105 \times$ pre-NCRT CD163 $+0.7772 \times$ pre-NCRT MCSF (Figure 4A). Using this formula, patients could be assigned a risk score that was associated with their individual

Table I Cox Regression Analysis of Macrophage Related Markers for Disease-Free Survival in Patients with LARC Following NCRT ( $\mathrm{n}=$ |9 I)

\begin{tabular}{|c|c|c|c|c|c|c|}
\hline \multirow[t]{2}{*}{ Variables } & \multicolumn{3}{|c|}{ Univariate Analysis } & \multicolumn{3}{|c|}{ Multivariate Analysis } \\
\hline & HR & $95 \% \mathrm{Cl}$ & $\mathbf{P}$ value & HR & $95 \% \mathrm{Cl}$ & $P$ value \\
\hline Pre-NCRT CDI 63 & I.0II & $1.008-1.015$ & $<0.001$ & 1.008 & $1.003-1.013$ & 0.003 \\
\hline Pre-NCRT MCSF & 2.878 & $1.826-4.534$ & $<0.001$ & 2.187 & $1.343-3.564$ & 0.002 \\
\hline Pre-NCRT CCL2 & $\mathrm{I} .440$ & $0.946-2.191$ & 0.089 & & & \\
\hline Pre-NCRT CD68 & 1.008 & $1.005-1.011$ & $<0.001$ & 1.002 & $0.997-1.006$ & 0.445 \\
\hline Post-surgery CDI63 & 1.004 & $1.001-1.006$ & 0.002 & 1.000 & $0.997-1.008$ & 0.377 \\
\hline Post-surgery MCSF & 1.702 & I.192-2.432 & 0.003 & 1.267 & $0.902-1.779$ & 0.172 \\
\hline Post-surgery CCL2 & 1.798 & $|.258-2.57|$ & 0.001 & $\mathrm{I} .494$ & $1.017-2.194$ & 0.041 \\
\hline Post-surgery CD68 & 1.003 & $1.001-1.005$ & 0.005 & 0.999 & $0.995-1.004$ & 0.751 \\
\hline
\end{tabular}

Abbreviations: LARC, locally advanced rectal cancer; NCRT, neoadjuvant chemoradiotherapy; HR, hazard ratio; Cl, confidential interval; ASA, American Society of Anesthesiologists; AJCC, American Joint Committee on Cancer; CEA, Carcinoembryonic Antigen; CAI9-9, Carbohydrate Antigen 19-9; NLR, neutrophil-to-lymphocyte ratio; SII, systemic immune-inflammation index; MLR, monocyte-to-lymphocyte ratio; PLR, platelet-to-lymphocyte ratio.

Table 2 Cox Regression Analysis of Macrophage Related Markers for Overall Survival in Patients with LARC Following NCRT ( $\mathrm{n}=19 \mathrm{I}$ )

\begin{tabular}{|l|c|c|c|c|c|c|}
\hline \multirow{2}{*}{ Variables } & \multicolumn{3}{|c|}{ Univariate Analysis } & \multicolumn{3}{c|}{ Multivariate Analysis } \\
\cline { 2 - 7 } & HR & $95 \%$ CI & P value & HR & $95 \%$ CI & P value \\
\hline Pre-NCRT CDI63 & 1.010 & $1.007-1.013$ & $<0.001$ & 1.005 & $1.001-1.011$ & 0.049 \\
Pre-NCRT MCSF & 3.372 & $1.987-5.724$ & $<0.001$ & 2.445 & $1.389-4.303$ & 0.002 \\
Pre-NCRT CCL2 & 1.644 & $1.018-2.654$ & 0.042 & 1.081 & $0.655-1.783$ & 0.761 \\
Pre-NCRT CD68 & 1.008 & $1.005-1.012$ & $<0.001$ & 1.003 & $0.998-1.008$ & 0.256 \\
Post-surgery CDI63 & 1.004 & $1.002-1.007$ & 0.001 & 1.001 & $0.995-1.008$ & 0.692 \\
Post-surgery MCSF & 1.509 & $1.014-2.245$ & 0.042 & 1.079 & $0.735-1.585$ & 0.697 \\
Post-surgery CCL2 & 1.696 & $1.138-2.529$ & 0.010 & 1.354 & $0.865-2.119$ & 0.185 \\
Post-surgery CD68 & 1.004 & $1.002-1.006$ & 0.001 & 1.001 & $0.996-1.007$ & 0.680 \\
\hline
\end{tabular}

Abbreviations: LARC, locally advanced rectal cancer; NCRT, neoadjuvant chemoradiotherapy; HR, hazard ratio; Cl, confidential interval. 


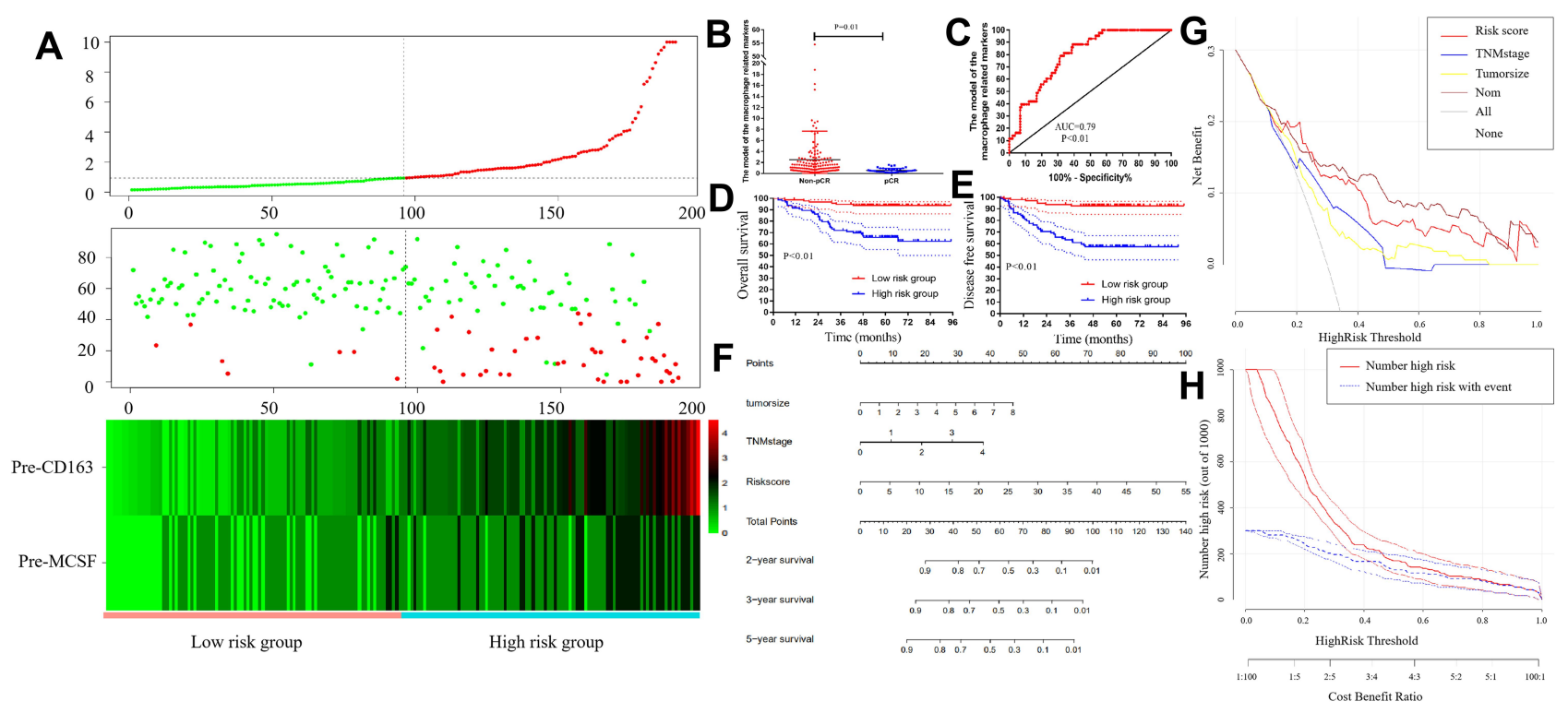

Figure 4 Construction of the risk factor model. (A) Risk factor model and training dataset. (Upper) Risk score distribution of I9I LARC patients. (Middle) Status of every patient in the external dataset $(\mathrm{N}=191)$. (Lower) Expression heatmap of the risk factors corresponding to each sample above. Red: high expression; Blue: low expression. (B) The risk scores were lower in the $P C R$ group when compared with the non-pCR group $(2.49 \pm 0.43$ vs $0.55 \pm 0.06, P<0.01)$. (C) ROC analysis demonstrating that the risk score has a powerful ability to predict $P C R(A \cup C=0.79, P<0.01)$. ( $D$ and $\mathbf{E})$ Kaplan-Meier analysis of disease-free survival and overall survival based on risk score models. $(\mathbf{F})$ Nomogram for the prediction of disease-free survival. (G) Decision curve analysis for pCR. (H) Clinical impact curve of the risk model. In I000 patients, the red solid line shows the total number of patients deemed to be at high risk for each risk threshold. The blue dashed line shows how many of those would be true positives.

prognosis. Using a cutoff value of 0.94 for risk scores generated from ROC curves, patients were divided into high-risk and low-risk groups. Patients in the low-risk group showed better DFS and OS than those in the high-risk group (all $P<0.001$, Figure 4D and E). Moreover, the risk score was also lower in the pCR group when compared with the nonpCR group $(P<0.01$, Figure 4B). ROC analysis demonstrated that the risk score had a powerful ability to predict $\mathrm{pCR}$ in LARC patients following NCRT $(\mathrm{AUC}=0.79, \quad P<0.01$, Figure 4C).

\section{Association of Risk Score with Patient Characteristics}

A total of 96 patients $(50.3 \%)$ were classified into the lowrisk score group and 95 patients (49.7\%) in the high-risk score group. Higher pre-NCRT CEA levels were found in the high-risk group $(P<0.05)$. No statistical differences were observed between the two groups in terms of gender, age, American Society of Anaesthesiology (ASA) grade, interval between NCRT and surgery, distance from the anal verge, clinical $\mathrm{T}$ stage, or clinical $\mathrm{N}$ stage, as shown in Table 3.

\section{Association of Risk Score with Perioperative Clinicopathological Parameters}

No significant differences were observed between the groups in terms of surgery duration, surgical approach,
peri-NCRT complications, peri-NCRT major complications or organ preservation procedures (Table 4). With regard to postoperative complications, no significant differences were found between the two groups in terms of postoperative hospital stay or postoperative complications ( $P=0.709, P=0.109$, respectively).

Compared with the low-risk group, the high-risk group was associated with worse pathological TNM and AJCC TRG stages (all $P<0.001$ ). Moreover, the tumor size was larger in the high-risk group $(2.5 \pm 1.3$ vs $3.2 \pm 1.5$, $P<0.001)$. The high-risk group showed significantly lower pCR rates than the low-risk group $(P<0.001)$. The pathological type, histopathological characteristics, degree of tumor differentiation, number of lymph nodes retrieved, and metastatic lymph nodes, were similar in both groups ( $P=0.26, P=0.374, P=0.391, P=0.197, P=0.293$, respectively). Similarly, neural invasion, CRM involvement, DRM involvement, and vascular invasion did not differ between the groups $(P=1.000, \quad P=1.000, \quad P=0.246$, $P=0.497$, respectively).

\section{Prognostic Factors for Disease-Free Survival and Overall Survival}

According to the univariate analysis, tumor size $(\mathrm{HR}=1.497$, $P<0.001)$, higher pathological TNM stage $(\mathrm{HR}=2.137$, $P<0.001$, AJCC TRG grade $(\mathrm{HR}=1.568, P=0.016)$, DRM 
Table 3 Baseline Characteristics in Patients with LARC Following NCRT Stratified by Risk Group of Macrophage Related Markers

\begin{tabular}{|c|c|c|c|}
\hline Characteristics & $\begin{array}{c}\text { Low Risk } \\
\text { Group } \\
(n=96)\end{array}$ & $\begin{array}{c}\text { High Risk } \\
\text { Group } \\
(n=95)\end{array}$ & $P$ value \\
\hline Sex (\%) & & & 0.092 \\
\hline Male & $58(60.4)$ & $69(72.6)$ & \\
\hline Female & $38(39.6)$ & $26(27.4)$ & \\
\hline Age (years) & $57.7 \pm 11.8$ & $56.1 \pm 12.6$ & 0.382 \\
\hline ASA score (\%) & & & 0.821 \\
\hline I & $67(69.8)$ & 70 (73.7) & \\
\hline 2 & $25(26.2)$ & $22(23.2)$ & \\
\hline 3 & $4(4.2)$ & $3(3.2)$ & \\
\hline $\begin{array}{l}\text { Distance from the anal } \\
\text { verge }(\mathrm{cm})\end{array}$ & $6.4 \pm 2.5$ & $6.8 \pm 2.7$ & 0.292 \\
\hline $\begin{array}{l}\text { Interval time between } \\
\text { NCRT and surgery } \\
\text { (weeks) }\end{array}$ & $8.7 \pm 1.9$ & $8.6 \pm 1.9$ & 0.720 \\
\hline Pre-NCRT cT stage (\%) & & & 0.938 \\
\hline $\mathrm{T} 2$ & $2(2.1)$ & $2(2.1)$ & \\
\hline T3 & $37(38.5)$ & $39(4 I .1)$ & \\
\hline $\mathrm{T} 4$ & $57(59.4)$ & $54(56.8)$ & \\
\hline Pre-NCRT cN stage (\%) & & & 1.000 \\
\hline No & $9(9.4)$ & $8(8.4)$ & \\
\hline $\mathrm{N}+$ & $87(90.6)$ & $87(91.6)$ & \\
\hline Pre-NCRT CEA (\%) & & & 0.007 \\
\hline$<5.0 \mathrm{ng} / \mathrm{mL}$ & 81 (84.4) & $64(67.4)$ & \\
\hline$\geq 5.0 \mathrm{ng} / \mathrm{mL}$ & $15(15.6)$ & $31(32.6)$ & \\
\hline
\end{tabular}

Abbreviations: NLR, neutrophil-to-lymphocyte ratio; LARC, locally advanced rectal cancer; NCRT, neoadjuvant chemoradiotherapy; ASA, American Society of Anesthesiologists; CEA, carcino embryonic antigen.

involvement $(\mathrm{HR}=10.170, P=0.027)$, pre-NCRT CEA level $(\mathrm{HR}=2.955, P=0.001)$, high risk score $(\mathrm{HR}=1.066, P<0.001)$, neural invasion $(\mathrm{HR}=3.418, P=0.020)$, and tumor differentiation $(\mathrm{HR}=2.271, P=0.040)$ were independently associated with disease free survival in LARC patients following NCRT. Cox regression analysis demonstrated that tumor size (HR=1.291, $P=0.041$ ), higher pathological TNM stage $(\mathrm{HR}=1.789, P=0.005)$, and high risk score $(\mathrm{HR}=1.084$, $P<0.001)$ remained significantly associated with impaired disease free survival, as shown in Table 5 .

According to the univariate analysis, tumor size (HR=1.462, $P<0.001$ ), higher pathological TNM stage (HR=1.847, $P<0.001$, AJCC TRG grade $(\mathrm{HR}=1.534$, $P=0.010)$, pre-NCRT CEA level $(\mathrm{HR}=2.459, P=0.002)$, and high risk score $(\mathrm{HR}=1.089, \quad P<0.001)$ were independently associated with overall survival in LARC patients following NCRT. Cox regression analysis demonstrated that tumor size $(\mathrm{HR}=1.335, P=0.002)$, higher pathological TNM stage $(\mathrm{HR}=1.554, P=0.031)$, and high risk score $(\mathrm{HR}=1.101, P<0.001)$ remained significantly associated with impaired overall survival, as shown in Table 6.

\section{Nomogram and Decision Curve Analysis for Disease-Free Survival}

Based on the results of the multivariate analysis, a nomogram for predicting disease-free survival was developed, as shown in Figure 4F. By summing up the score of each variable, a straight line could be drawn to obtain the predicted 3-year DFS rate. The C-index of the nomogram was 0.76 (95\% CI 0.71-0.81).

Decision curve analysis (DCA) was used to evaluate the performance of the nomogram for predicting diseasefree survival. As shown in Figure 4G, the nomogram was better than either risk score, pathological TNM stage or tumor size for predicting disease-free survival. The clinical impact curve (Figure $4 \mathrm{H}$ ) shows the prediction of risk stratification in 1000 patients using a resampling bootstrap method. "Number high risk" indicates the number of patients classified as positive (high risk) by the risk score according to various threshold probabilities. "Number high risk with the event" indicates the true positive patient number according to various threshold probabilities.

\section{Discussion}

Macrophages and secreted molecules in the tumor microenvironment are important factors affecting the efficiency and toxicity of NCRT in patients with rectal cancer. To the best of our best knowledge, few studies have evaluated the clinical value of the combined assessment of macrophages and secreted molecules in the tumor micro-environment in LARC patients following NCRT. In the present study, we demonstrated that assessment of pre-/post-NCRT macrophage, TAM, CCL2, and MCSF1 scores can effectively predict the prognosis in LARC patients. Based on COX analysis, a risk score model was constructed which can predict prognosis and NCRT response in LARC patients. Finally, a nomogram was constructed to predict survival outcome.

The association between tumor-infiltrating lymphocytes and tumor biology was first noticed by Virchow in $1863 .^{28}$ During tumor development, a high density of 
Table 4 Operative and Postoperative Outcomes in Patients with LARC Following NCRT Stratified by Risk Group of Macrophage Related Markers

\begin{tabular}{|c|c|c|c|}
\hline Characteristics & Low Risk Group $(n=96)$ & High Risk Group $(n=95)$ & $P$ value \\
\hline Operative time (min) & $219.3 \pm 52.4$ & $218.2 \pm 55.5$ & 0.895 \\
\hline Pathological type (\%) & & & 0.226 \\
\hline Ulcering & $91(94.8)$ & $85(89.5)$ & \\
\hline Expanding & $3(3.1)$ & $3(3.2)$ & \\
\hline Infiltrating & $2(2.1)$ & $7(7.4)$ & \\
\hline Histopathology (\%) & & & 0.374 \\
\hline Adenocarcinoma & $87(90.6)$ & $82(86.3)$ & \\
\hline Mucinous or signet ring cell carcinoma & $9(9.4)$ & $13(13.7)$ & \\
\hline Tumor differentiation (\%) & & & 0.391 \\
\hline Well to moderately differentiated & $86(89.6)$ & $81(85.3)$ & \\
\hline Poorly differentiated and others & $10(10.4)$ & $14(14.7)$ & \\
\hline Postoperative hospital stay (days) & $9.0 \pm 5.8$ & $9.1 \pm 4.9$ & 0.882 \\
\hline Postoperative complications (\%) & $15(15.6)$ & $18(18.9)$ & 0.571 \\
\hline During CRT complication*(\%) & $42(43.8)$ & $45(47.4)$ & 0.664 \\
\hline Major & $4(4.2)$ & $\mathrm{I}(\mathrm{I} . \mathrm{I})$ & 0.368 \\
\hline Organ preservation (\%) & $86(89.6)$ & $87(91.6)$ & 0.805 \\
\hline Lymph nodes retrieved & $12.0 \pm 7.2$ & $13.7 \pm 10.2$ & 0.197 \\
\hline Metastatic lymph nodes & $0.8 \pm 4.0$ & $1.3 \pm 3.1$ & 0.293 \\
\hline CRM involvement (\%) & I (I.0) & $\mathrm{I}(\mathrm{I} . \mathrm{I})$ & 1.000 \\
\hline DRM involvement (\%) & $0(0.0)$ & $2(2.1)$ & 0.246 \\
\hline Tumor size $(\mathrm{cm})$ & $2.5 \pm 1.3$ & $3.2 \pm 1.5$ & $<0.001$ \\
\hline Pathological TNM stage (\%) & & & $<0.001$ \\
\hline 0 & $39(40.6)$ & $5(5.3)$ & \\
\hline 1 & $19(19.8)$ & $21(22.1)$ & \\
\hline II & $24(25.0)$ & $33(34.7)$ & \\
\hline III & $14(14.6)$ & $35(36.8)$ & \\
\hline IV & $0(0.0)$ & I (I.I) & \\
\hline TRG (\%) & & & $<0.001$ \\
\hline 0 & $39(40.6)$ & $5(5.3)$ & \\
\hline 1 & $22(22.9)$ & $31(32.6)$ & \\
\hline 2 & $31(32.3)$ & $49(51.6)$ & \\
\hline 3 & $4(4.2)$ & $10(10.5)$ & \\
\hline PCR rates $(\%)$ & $39(40.6)$ & $5(5.3)$ & $<0.001$ \\
\hline Nerval invasion (\%) & $4(4.2)$ & $4(4.2)$ & 1.000 \\
\hline Vascular invasion (\%) & $2(2.1)$ & $0(0.0)$ & 0.497 \\
\hline
\end{tabular}

Abbreviations: LARC, locally advanced rectal cancer; NCRT, neoadjuvant chemoradiotherapy; CRM, circumferential resection margin; DRM, distal resection margin; TRG, tumor regression grade; $\mathrm{PCR}$, pathological complete response.

infiltrating lymphocytes and macrophages can promote cell mutagenesis, proliferation and metastasis. ${ }^{29-32}$ Moreover, the molecules secreted by the tumor cells or TAMs play an important role modulating the immune response in the tumor micro-environment. ${ }^{33-38}$ In the present study, we analyzed the density of macrophages and TAMs in the tumor tissue before and after NCRT. Our results contradict a previous meta-analysis of 55 studies and 8692 patients which concluded that TAMs were correlated with survival. ${ }^{39}$ In most tumor types, such as breast, gastric, oral, ovarian, and bladder cancer, high TAM density correlates with worse overall survival. In contrast, in colorectal cancer (CRC) high TAM density was found to be associated with improved survival (5 
Table 5 Cox Regression Analysis of Predictive Factors for Overall Survival in Patients with LARC Following NCRT ( $\mathrm{n}=19 \mathrm{I})$

\begin{tabular}{|c|c|c|c|c|c|c|}
\hline \multirow[t]{2}{*}{ Variables } & \multicolumn{3}{|c|}{ Univariate Analysis } & \multicolumn{3}{|c|}{ Multivariate Analysis } \\
\hline & HR & $95 \% \mathrm{Cl}$ & $P$ value & HR & $95 \% \mathrm{Cl}$ & $P$ value \\
\hline Sex, male/female & 0.901 & $0.455-1.787$ & 0.766 & & & \\
\hline Age & 0.991 & $0.965-1.017$ & 0.486 & & & \\
\hline ASA & 0.930 & $0.511-1.692$ & 0.812 & & & \\
\hline Distance from the anal verge & 1.002 & $0.892-1.125$ & 0.977 & & & \\
\hline Tumor size & 1.497 & $1.223-1.833$ & $<0.001$ & 1.291 & $1.011-1.649$ & 0.041 \\
\hline Pathological TNM stage & 2.137 & $1.488-3.068$ & $<0.001$ & 1.789 & I.197-2.674 & 0.005 \\
\hline AJCC grade & 1.568 & $|.087-2.26|$ & 0.016 & 0.844 & $0.534-1.334$ & 0.468 \\
\hline $\begin{array}{l}\text { Interval time between NCRT and } \\
\text { surgery }\end{array}$ & 0.913 & $0.780-1.069$ & 0.260 & & & \\
\hline DRM involvement & 10.170 & I.297-79.742 & 0.027 & 5.322 & $0.602-47.035$ & 0.133 \\
\hline CRM involvement & 2.373 & $0.325-\mid 7.311$ & 0.394 & & & \\
\hline Pre-NCRT cT stage & 1.076 & $0.584-1.982$ & 0.814 & & & \\
\hline Pre-NCRT cN stage & 1.256 & $0.386-4.086$ & 0.705 & & & \\
\hline Postoperative hospital stay & 1.023 & $0.976-1.072$ & 0.339 & & & \\
\hline Pre-NCRT CEA level & 2.955 & $1.558-5.606$ & 0.001 & 1.971 & $0.99 \mid-3.922$ & 0.053 \\
\hline Risk score & 1.066 & $1.04|-1.09|$ & $<0.001$ & 1.084 & $1.053-1.117$ & $<0.001$ \\
\hline Postoperative complications & 1.182 & $0.494-2.827$ & 0.707 & & & \\
\hline Nerval invasion & 3.418 & $1.210-9.660$ & 0.020 & 1.972 & $0.663-5.864$ & 0.222 \\
\hline Vascular invasion & 0.512 & $0.188-3.398$ & 0.647 & & & \\
\hline Tumor differentiation & 2.271 & $1.040-4.957$ & 0.040 & 1.322 & $0.545-3.203$ & 0.537 \\
\hline Histopathology & & & 0.118 & & & \\
\hline Expanding & Reference & Reference & & & & \\
\hline Infiltrating & 3.457 & $1.059-11.288$ & 0.040 & & & \\
\hline Ulcering & 1.307 & $0.313-5.449$ & 0.713 & & & \\
\hline
\end{tabular}

Abbreviations: LARC, locally advanced rectal cancer; NCRT, neoadjuvant chemoradiotherapy; HR, hazard ratio; Cl, confidential interval; ASA, American Society of Anesthesiologists; AJCC, American Joint Committee on Cancer; CEA, carcinoembryonic antigen; CRM, circumferential resection margin; DRM, distal resection margin.

studies, 1149 patients). However, we noticed that the TAMs in the previous meta-analysis were identified based on the CD68 marker. The CD68 marker is a surface antigen expressed by both M1 and M2 subtype macrophages. In the tumor micro-environment, the M2 subtype of TAM can be distinguished by expression of the CD163 surface antigen. ${ }^{31,32,38}$ In the present study, we found that assessing both markers before and after NCRT could predict DFS and OS in LARC patients. This result, which is the opposite of that reported in the previously mentioned meta-analysis, may also be related to the fact that the patients included in the meta-analysis did not accept NCRT. Moreover, in the present study, the TAMs were identified based on CD163+, instead of generalized CD68+ staining.

CCL2 is an important chemoattractant that recruits monocytes from peripheral blood into cancerous tissues and which is expressed by tumor cells, macrophages, and stromal cells. TAMs can exert a suppressive effect within the tumor micro-environment. ${ }^{40-43}$ CCL2 secretion by
TAMs has also been detected and contributes to M2 polarization. $^{44}$ Moreover, several small molecule inhibitors based on the CCL2 receptor (CCR2), such as CCX872-B, PF-04136309, MLN1202, PF-04136309, and BMS813,160 , are currently in clinical trials for the treatment of solid tumors. MCSF, also known as CSF1, is a critical macrophage growth factor that plays an important role in monocyte generation and TAM activation. ${ }^{38}$ Growing evidence shows that TAMs can influence the outcome of cancer chemotherapy and radiotherapy resistance. ${ }^{45-48}$ However, there are no relevant studies evaluating the predictive ability of CCL2 and MCSF levels in LARC patients. In the present study, we analyzed the clinical value of CCL2 and MCSF for the prediction of NCRT response and prognosis in LARC patients. The results demonstrated that CCL2 showed better predictive ability than MCSF. Moreover, assessment of CCL2 expression was more accurate before NCRT than after NCRT. This may be due to the fact that NCRT suppresses the immune response systemically and in the tumor micro-environment. In the present 
Table 6 Cox Regression Analysis of Predictive Factors for Disease-Free Survival in Patients with LARC Following NCRT ( $\mathrm{n}=19 \mathrm{I})$

\begin{tabular}{|c|c|c|c|c|c|c|}
\hline \multirow[t]{2}{*}{ Variables } & \multicolumn{3}{|c|}{ Univariate Analysis } & \multicolumn{3}{|c|}{ Multivariate Analysis } \\
\hline & HR & $95 \% \mathrm{Cl}$ & $P$ value & HR & $95 \% \mathrm{Cl}$ & $P$ value \\
\hline Sex, male/female & 0.756 & $0.407-1.406$ & 0.377 & & & \\
\hline Age & 1.003 & $0.980-1.027$ & 0.798 & & & \\
\hline ASA & 0.979 & $0.585-1.639$ & 0.937 & & & \\
\hline Distance from the anal verge & 1.000 & $0.901-1.109$ & 0.993 & & & \\
\hline Tumor size & 1.462 & I.229-1.739 & $<0.001$ & 1.335 & I.107-1.608 & 0.002 \\
\hline Pathological TNM stage & 1.847 & $1.374-2.483$ & $<0.001$ & 1.554 & $1.097-2.200$ & 0.013 \\
\hline AJCC grade & 1.534 & $1.109-2.121$ & 0.010 & 0.991 & $0.666-1.474$ & 0.965 \\
\hline Interval time between NCRT and surgery & 0.949 & $0.824-1.093$ & 0.466 & & & \\
\hline DRM involvement & 6.752 & $0.907-50.276$ & 0.062 & & & \\
\hline CRM involvement & 1.998 & $0.276-14.488$ & 0.494 & & & \\
\hline Pre-NCRT cT stage & 1.164 & $0.68 I-1.989$ & 0.578 & & & \\
\hline Pre-NCRT cN stage & 0.657 & $0.279-1.543$ & 0.335 & & & \\
\hline Postoperative hospital stay & 1.026 & $0.984-1.070$ & 0.229 & & & \\
\hline Pre-NCRT CEA level & 2.459 & $1.389-4.353$ & 0.002 & 1.575 & $0.859-2.890$ & 0.142 \\
\hline Risk score & 1.089 & $1.061-1.118$ & $<0.001$ & 1.101 & $1.069-1.134$ & $<0.001$ \\
\hline Postoperative complications & 0.991 & $0.691-1.423$ & 0.963 & & & \\
\hline Nerval invasion & 2.363 & $0.849-6.582$ & 0.100 & & & \\
\hline Vascular invasion & 0.689 & $0.324-2.105$ & 0.602 & & & \\
\hline Tumor differentiation & 1.582 & $0.74 I-3.377$ & 0.236 & & & \\
\hline Histopathology & & & 0.209 & & & \\
\hline Expanding & Reference & Reference & & & & \\
\hline Infiltrating & 2.761 & $0.856-8.904$ & 0.089 & & & \\
\hline Ulcering & 1.430 & $0.443-4.610$ & 0.550 & & & \\
\hline
\end{tabular}

Abbreviations: LARC, locally advanced rectal cancer; NCRT, neoadjuvant chemoradiotherapy; HR, hazard ratio; Cl, confidential interval; ASA, American Society of Anesthesiologists; AJCC, American Joint Committee on Cancer; CEA, carcinoembryonic antigen; CRM, circumferential resection margin; DRM, distal resection margin.

study, we constructed our model based on expression before NCRT.

Our goal was to select the best possible marker or macrophage-associated factor which could be used to predict DFS and OS in LARC patients. Multiple COX analysis demonstrated that the density of M2 subtype (CD163+ macrophages) and CCL2 expression levels were independent factors which could be used to predict DFS and OS in LARC patients. Based on the previous result, we constructed a risk factor model to calculate a risk score for each patient. Risk score models have been found to possess a powerful ability to predict prognosis in several cancer types. ${ }^{26,49}$ However, to the best of our knowledge, there have been no studies describing risk score models based on macrophageassociated factors to predict NCRT response and prognosis in LARC patients following TME surgery. In the present study, the model not only showed a powerful ability to predict prognosis, but also accurately predicted $\mathrm{pCR}$. Summing up, in the present study we constructed an effective model to predict prognosis and NCRT response in LARC patients.
To explore the efficiency of the model in clinical practice, we divided the patients in half based on the risk score. A total of 7 patients experienced disease recurrence in the low risk score group, whereas 42 patients did so in the high risk score group. Moreover, LARC patients who underwent NCRT and achieved pCR showed a better response and prognosis. Previous studies uncovered several factors which can be used to predict pCR. However, these predictive factors are almost entirely based on clinical results after NCRT. Currently there is no way of predicting the NCRT response before NCRT. In the present study, the risk score model was based on results from the biopsy, before NCRT. Thus, the model can be used to predict the outcome of NCRT and to exclude patients who may not respond to this procedure but who may suffer from NCRT complications.

This study has several limitations that warrant discussion. First, there could be potential selection bias due to its retrospective design. Second, the impact of the systematic inflammatory response was not assessed due to lack of complete 
medical records. Notwithstanding these limitations, we believe this study contributes to our understanding of the impact of systemic inflammation on oncological outcomes in patients with LARC following NCRT.

In conclusion, high TAMs and associated factors in the tumor micro-environment were associated with poorer DFS and OS in LARC patients. In addition, M2 subtype $(\mathrm{CD} 163+)$ macrophages and CCL2 expression were identified as the most effective markers, and their prognostic value was further confirmed by KM analysis. Finally, a risk factor model was constructed to help predict survival. More intensive adjuvant treatment could be considered following NCRT for patients with LARC and higher risk factor scores. Large scale prospective clinical trials are warranted to confirm the previous findings.

\section{Ethics Approval and Consent to Participate}

The study was conducted in accordance with the Declaration of Helsinki, and the protocol was approved by the Ethics Committee of Fujian Medical University Union Hospital (2013051). And all patients provided written informed consent for the scientific use of the clinical tissue samples and clinical data.

\section{Funding}

This study was supported by the Science Foundation of the Fujian Province, (No. 2016J01602; 2019J0105), Special Financial Foundation of Fujian Provincial (No. 2020B019; No. 2015-1297), Young and middle-aged backbone training project in the health system of Fujian province (2015-ZQN-JC-17; 2016-ZQN-26), the Startup Fund for Scientific Research, Fujian Medical University (2018QH2027) and Professor Development Foundation of Fujian Medical University (No. JS11006).

\section{Disclosure}

The authors declared that they have no conflicts of interest for this work.

\section{References}

1. Heusinkveld M, van der Burg SH. Identification and manipulation of tumor associated macrophages in human cancers. $J$ Transl Med. 2011;9(1):216. doi:10.1186/1479-5876-9-216

2. Bingle L, Brown NJ, Lewis CE. The role of tumour-associated macrophages in tumour progression: implications for new anticancer therapies. J Pathol. 2002;196(3):254-265. doi:10.1002/path.1027

3. Siveen KS, Kuttan G. Role of macrophages in tumour progression. Immunol Lett. 2009;123(2):97-102. doi:10.1016/j.imlet.2009.02.011
4. Jeannin P, Paolini L, Adam C, Delneste Y. The roles of CSFs on the functional polarization of tumor-associated macrophages. FEBS $J$. 2018;285(4):680-699. doi:10.1111/febs.14343

5. Sica A, Schioppa T, Mantovani A, Allavena P. Tumour-associated macrophages are a distinct M2 polarised population promoting tumour progression: potential targets of anti-cancer therapy. Eur $J$ Cancer. 2006;42(6):717-727. doi:10.1016/j.ejca.2006.01.003

6. Martínez VG, Rubio C, Martínez-Fernández M, et al. BMP4 induces M2 macrophage polarization and favors tumor progression in bladder cancer. Clin Cancer Res. 2017;23(23):7388-7399. doi:10.1158/10780432.CCR-17-1004

7. Yeung OW, Lo CM, Ling CC, et al. Alternatively activated (M2) macrophages promote tumour growth and invasiveness in hepatocellular carcinoma. J Hepatol. 2015;62(3):607-616. doi:10.1016/j. jhep.2014.10.029

8. Ohashi T, Aoki M, Tomita H, et al. M2-like macrophage polarization in high lactic acid-producing head and neck cancer. Cancer Sci. 2017;108(6):1128-1134. doi:10.1111/cas.13244

9. Choi J, Gyamfi J, Jang H, Koo JS. The role of tumor-associated macrophage in breast cancer biology. Histol Histopathol. 2018;33 (2):133-145. doi:10.14670/HH-11-916

10. Korehisa S, Oki E, Iimori $\mathrm{M}$, et al. Clinical significance of programmed cell death-ligand 1 expression and the immune microenvironment at the invasive front of colorectal cancers with high microsatellite instability. Int $J$ Cancer. 2018;142(4):822-832. doi:10.1002/ijc.31107

11. Komohara Y, Fujiwara Y, Ohnishi K, Takeya M. Tumor-associated macrophages: potential therapeutic targets for anti-cancer therapy. Adv Drug Deliv Rev. 2016;99(Pt B):180-185. doi:10.1016/j. addr.2015.11.009

12. Qian BZ, Pollard JW. Macrophage diversity enhances tumor progression and metastasis. Cell. 2010;141(1):39-51. doi:10.1016/j. cell.2010.03.014

13. Kogure A, Kosaka N, Ochiya T. Cross-talk between cancer cells and their neighbors via miRNA in extracellular vesicles: an emerging player in cancer metastasis. $J$ Biomed Sci. 2019;26(1):7. doi:10.1186/s12929-019-0500-6

14. Movahedi K, Van Ginderachter JA, Ontogeny T. Microenvironmental regulation of tumor-associated macrophages. Antioxid Redox Signal. 2016;25(14):775-791. doi:10.1089/ars.2016.6704

15. Roh MS, Colangelo LH, O'Connell MJ, et al. Preoperative multimodality therapy improves disease-free survival in patients with carcinoma of the rectum: NSABP R-03. J Clin Oncol. 2009;27 (31):5124-5130. doi:10.1200/JCO.2009.22.0467

16. Sauer R, Liersch T, Merkel S, et al. Preoperative versus postoperative chemoradiotherapy for locally advanced rectal cancer: results of the German CAO/ARO/AIO-94 randomized Phase III trial after a median follow-up of 11 years. J Clin Oncol. 2012;30(16):1926-1933. doi:10.1200/JCO.2011.40.1836

17. van Gijn W, Marijnen CA, Nagtegaal ID, et al. Preoperative radiotherapy combined with total mesorectal excision for resectable rectal cancer: 12-year follow-up of the multicentre, randomised controlled TME trial. Lancet Oncol. 2011;12(6):575-582. doi:10.1016/S14702045(11)70097-3

18. Ha YJ, Tak KH, Kim CW, et al. PSMB8 as a candidate marker of responsiveness to preoperative radiation therapy in rectal cancer patients. Int J Radiat Oncol Biol Phys. 2017;98(5):1164-1173. doi:10.1016/j.ijrobp.2017.03.023

19. Figueiredo P, Lepland A, Scodeller P, et al. Peptide-guided resiquimod-loaded lignin nanoparticles convert tumor-associated macrophages from M2 to M1 phenotype for enhanced chemotherapy. Acta Biomater. 2020. doi:10.1016/j.actbio.2020.09.038

20. Kim SH, Saeidi S, Zhong X, et al. Breast cancer cell debris diminishes therapeutic efficacy through heme oxygenase-1-mediated inactivation of M1-like tumor-associated macrophages. Neoplasia. 2020;22(11):606-616. doi:10.1016/j.neo.2020.08.006 
21. Mantovani A, Marchesi F, Malesci A, Laghi L, Allavena P. Tumourassociated macrophages as treatment targets in oncology. Nat Rev Clin Oncol. 2017;14(7):399-416. doi:10.1038/nrclinonc.2016.217

22. Genard G, Lucas S, Michiels C. Reprogramming of tumor-associated macrophages with anticancer therapies: radiotherapy versus chemoand immunotherapies. Front Immunol. 2017;8:828. doi:10.3389/ fimmu.2017.00828

23. Zhang Y, Liu X, Xu M, Chen K, Li S, Guan G. Prognostic value of pretreatment systemic inflammatory markers in patients with locally advanced rectal cancer following neoadjuvant chemoradiotherapy. Sci Rep. 2020;10(1):8017. doi:10.1038/s41598-020-64684-z

24. Ryan R, Gibbons D, Hyland JM, et al. Pathological response following long-course neoadjuvant chemoradiotherapy for locally advanced rectal cancer. Histopathology. 2005;47(2):141-146. doi:10.1111/ j.1365-2559.2005.02176.x

25. Zhang Y, Xu Z, Sun Y, Chi P, Lu X. Knockdown of KLK11 reverses oxaliplatin resistance by inhibiting proliferation and activating apoptosis via suppressing the PI3K/AKT signal pathway in colorectal cancer cell. Onco Targets Ther. 2018;11:809-821. doi:10.2147/OTT. S151867

26. Zhang Y, Xu M, Chen J, et al. Prognostic value of the FOXK family expression in patients with locally advanced rectal cancer following neoadjuvant chemoradiotherapy. Onco Targets Ther. 2020;13:91 85-9201. doi:10.2147/OTT.S255956

27. Camp RL, Dolled-Filhart M, Rimm DL. X-tile: a new bio-informatics tool for biomarker assessment and outcome-based cut-point optimization. Clin Cancer Res. 2004;10(21):7252-7259. doi:10.11 58/1078-0432.CCR-04-0713

28. Balkwill F, Mantovani A. Inflammation and cancer: back to Virchow. Lancet. 2001;357(9255):539-545. doi:10.1016/S0140-6736(00)04046-0

29. Wang HW, Joyce JA. Alternative activation of tumor-associated macrophages by IL-4: priming for protumoral functions. Cell Cycle. 2010;9(24):4824-4835. doi:10.4161/cc.9.24.14322

30. Caux C, Ramos RN, Prendergast GC, Bendriss-Vermare N, Ménétrier-Caux C, Milestone A. Review on how macrophages affect tumor growth. Cancer Res. 2016;76(22):6439-6442. doi:10.1158/ 0008-5472.CAN-16-2631

31. Ngambenjawong C, Gustafson HH, Pun SH. Progress in tumor-associated macrophage (TAM)-targeted therapeutics. $A d v$ Drug Deliv Rev. 2017;114:206-221. doi:10.1016/j.addr.2017.04.010

32. Quail DF, Joyce JA. Microenvironmental regulation of tumor progression and metastasis. Nat Med. 2013;19(11):1423-1437. doi:10.1038/nm.3394

33. Qian BZ, Li J, Zhang H, et al. CCL2 recruits inflammatory monocytes to facilitate breast-tumour metastasis. Nature. 2011;475 (7355):222-225. doi:10.1038/nature10138

34. Escamilla J, Schokrpur S, Liu C, et al. CSF1 receptor targeting in prostate cancer reverses macrophage-mediated resistance to androgen blockade therapy. Cancer Res. 2015;75(6):950-962. doi:10.1158/ 0008-5472.CAN-14-0992

35. Shiao SL, Ruffell B, DeNardo DG, Faddegon BA, Park CC, Coussens LM. TH2-polarized CD4(+) $\mathrm{T}$ cells and macrophages limit efficacy of radiotherapy. Cancer Immunol Res. 2015;3 (5):518-525. doi:10.1158/2326-6066.CIR-14-0232

OncoTargets and Therapy

\section{Publish your work in this journal}

OncoTargets and Therapy is an international, peer-reviewed, open access journal focusing on the pathological basis of all cancers, potential targets for therapy and treatment protocols employed to improve the management of cancer patients. The journal also focuses on the impact of management programs and new therapeutic

Submit your manuscript here: https://www.dovepress.com/oncotargets-and-therapy-journal
36. Paulus P, Stanley ER, Schäfer R, Abraham D, Aharinejad S. Colonystimulating factor-1 antibody reverses chemoresistance in human MCF-7 breast cancer xenografts. Cancer Res. 2006;66 (8):4349-4356. doi:10.1158/0008-5472.CAN-05-3523

37. Xu J, Escamilla J, Mok S, et al. CSF1R signaling blockade stanches tumor-infiltrating myeloid cells and improves the efficacy of radiotherapy in prostate cancer. Cancer Res. 2013;73(9):2782-2794. doi:10.1158/0008-5472.CAN-12-3981

38. Chen Y, Song Y, Du W, Gong L, Chang H, Zou Z. Tumor-associated macrophages: an accomplice in solid tumor progression. $J$ Biomed Sci. 2019;26(1):78. doi:10.1186/s12929-019-0568-z

39. Zhang QW, Liu L, Gong CY, et al. Prognostic significance of tumor-associated macrophages in solid tumor: a meta-analysis of the literature. PLoS One. 2012;7(12):e50946. doi:10.1371/journal. pone. 0050946

40. Cai Z, Chen Q, Chen J, et al. Monocyte chemotactic protein 1 promotes lung cancer-induced bone resorptive lesions in vivo. Neoplasia. 2009;11(3):228-236. doi:10.1593/neo.81282

41. Lesokhin AM, Hohl TM, Kitano S, et al. Monocytic CCR2(+) myeloid-derived suppressor cells promote immune escape by limiting activated CD8 T-cell infiltration into the tumor microenvironment. Cancer Res. 2012;72(4):876-886. doi:10.1158/0008-5472.CAN-11-1792

42. Lu Y, Cai Z, Galson DL, et al. Monocyte chemotactic protein-1 (MCP-1) acts as a paracrine and autocrine factor for prostate cancer growth and invasion. Prostate. 2006;66(12):1311-1318. doi:10.1002/ pros. 20464

43. Ueno T, Toi M, Saji H, et al. Significance of macrophage chemoattractant protein-1 in macrophage recruitment, angiogenesis, and survival in human breast cancer. Clin Cancer Res. 2000;6 (8):3282-3289.

44. Balkwill F. Cancer and the chemokine network. Nat Rev Cancer. 2004;4(7):540-550. doi:10.1038/nrc1388

45. Goulielmaki E, Bermudez-Brito $M$, Andreou $M$, et al. Pharmacological inactivation of the PI3K p $110 \delta$ prevents breast tumour progression by targeting cancer cells and macrophages. Cell Death Dis. 2018;9(6):678. doi:10.1038/s41419-018-0717-4

46. Pyonteck SM, Gadea BB, Wang HW, et al. Deficiency of the macrophage growth factor CSF-1 disrupts pancreatic neuroendocrine tumor development. Oncogene. 2012;31(11):1459-1467. doi:10.1038/onc. 2011.337

47. Cai H, Zhu XD, Ao JY, et al. Colony-stimulating factor-1-induced AIF1 expression in tumor-associated macrophages enhances the progression of hepatocellular carcinoma. Oncoimmunology. 2017;6(9): e1333213. doi:10.1080/2162402X.2017.1333213

48. Zhu Y, Knolhoff BL, Meyer MA, et al. CSF1/CSF1R blockade reprograms tumor-infiltrating macrophages and improves response to T-cell checkpoint immunotherapy in pancreatic cancer models. Cancer Res. 2014;74(18):5057-5069. doi:10.1158/0008-5472.CAN13-3723

49. Kivipelto M, Ngandu T, Laatikainen T, Winblad B, Soininen H, Tuomilehto J. Risk score for the prediction of dementia risk in 20 years among middle aged people: a longitudinal, population-based study. Lancet Neurol. 2006;5(9):735-741. doi:10.1016/S1474-4422(06)70537-3

agents and protocols on patient perspectives such as quality of life, adherence and satisfaction. The manuscript management system is completely online and includes a very quick and fair peer-review system, which is all easy to use. Visit http://www.dovepress.com/ testimonials.php to read real quotes from published authors. 